\title{
Nanoparticles and Cellular Health
}

Jeffrey G. Ault, Alvaro Mendoza, Joan Pedersen-Lane, Dong Hong Gao, Samuel S. Bowser, Jane Kasten-Jolly, and David, A. Lawrence

The Wadsworth Center, New York State Department of Health, Empire State Plaza, Albany, NY 12201-0509

Like those for asbestos in the 1950s and 1960s, the uses for nanoparticles appear endless. Nanotechnology is touted as the new driver of a future economy. However, before the dependency on various kinds of nanoparticles becomes too great, their individual impact on human health needs to be addressed to avoid the public health dilemma that followed the increased use of asbestos. Like with asbestos, size and dimensions as well as chemical composition may affect the toxicity of nanoparticles.

The aim of our study was to assess the cytotoxic effects in vitro of silica nanoparticles on human peripheral blood mononuclear cells (PBMCs). PBMCs were cultured in medium with varying concentrations of $10-\mathrm{nm}$ or $100-\mathrm{nm}$ silica beads for 24 hours. Cytotoxic effects were measured by screening for indicators of oxidative stress including glutathione (GSH) depletion, the generation of radical species, and pro-inflammatory cytokine responses. Flow cytometry was used to measure cell viability and surface thiol levels after nanoparticle exposure. Nanoparticle behavior outside and inside the cell was observed with electron microscopy, while immunoelectron microscopy was used to investigate GSH distribution in nanoparticle-exposed cells. GSH is the most abundant of the lowmolecular-mass molecules that provide reducing equivalents that protect cells from oxidative stress. As its role as protector implies, GSH is found distributed relatively evenly throughout the cell, with lesser amounts in the endoplasmic reticulum where protein folding occurs [1].

We found at higher concentrations, particularly with the 10-nm particles, nanoparticles can induce cytokine inflammatory responses and oxidative stress in cells in vitro. Cellular GSH distribution in nanoparticle-exposed cells was similar to that found in oxidatively stressed cells [1] in that GSH was being depleted from the cell and the last GSH reserves were located in the mitochondria (Fig. 1).

Asbestos and other mineral fibers have been called "physical carcinogens" because a fiber's aspect ratio is important in determining its carcinogenic potential. Their size and shape are as important as their chemical composition in their ability to induce inflammation and oxidative stress as well as disruption of genetic material and its equal distribution during cell division [2 and references within]. When investigating the harmful effects of nanoparticles, one needs to keep in mind that size and shape may be as important as chemical content. We have shown this to be true in this study. The two classes of beads were of the same chemical composition, but of different size. The 10-nm silica particles were found to be more toxic than the $100-\mathrm{nm}$ ones at lower concentrations [3].

\section{References}

[1] J. G. Ault and D. A. Lawrence, Exp. Cell Res. 285 (2003) 9-14.

[2] J. G. Ault et al., Cancer Res. 55 (1995) 792-798.

[3] This work was done at the Wadsworth Center's EM Core and Immunology Core facilities. 


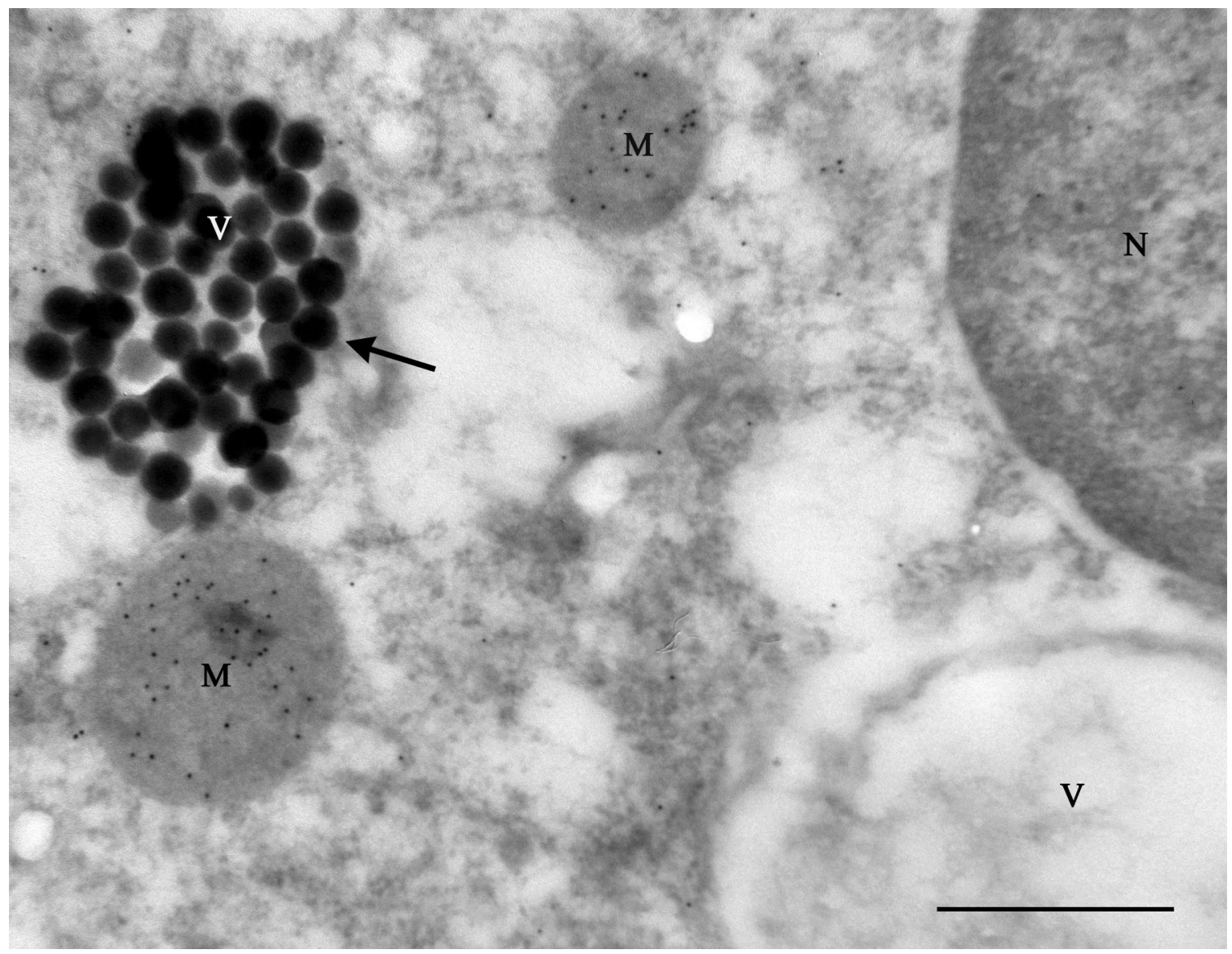

Fig. 1. GSH distribution in a cell exposed to 100-nm silica particles. The silica particles (arrow) are located in a vacuole (V), presumably either a phagosome or phagolysosome. GSH (indicated by 10$\mathrm{nm}$ gold particles) is mostly localized in mitochondria (M) and not in the nucleus $(\mathrm{N})$ or cytoplasm. Bar equals 0.5 micron. 